\title{
A 2D SYSTEM APPROACH TO THE DESIGN OF A ROBUST MODIFIED REPETITIVE-CONTROL SYSTEM WITH A DYNAMIC OUTPUT-FEEDBACK CONTROLLER
}

\author{
LAN ZHOU *, JinhUA SHE **, ShaOWU ZHOU * \\ ${ }^{*}$ School of Information and Electrical Engineering \\ Hunan University of Science and Technology, Xiangtan 411201, China \\ e-mail: zhoulan@hnust.edu.cn \\ ${ }^{* *}$ School of Computer Science \\ Tokyo University of Technology, Tokyo 192-0982, Japan
}

\begin{abstract}
This paper is concerned with the problem of designing a robust modified repetitive-control system with a dynamic outputfeedback controller for a class of strictly proper plants. Employing the continuous lifting technique, a continuous-discrete two-dimensional (2D) model is built that accurately describes the features of repetitive control. The 2D control input contains the direct sum of the effects of control and learning, which allows us to adjust control and learning preferentially. The singular-value decomposition of the output matrix and Lyapunov stability theory are used to derive an asymptotic stability condition based on a Linear Matrix Inequality (LMI). Two tuning parameters in the LMI manipulate the preferential adjustment of control and learning. A numerical example illustrates the tuning procedure and demonstrates the effectiveness of the method.
\end{abstract}

Keywords: repetitive control, dynamic output-feedback, two-dimensional system, singular-value decomposition, linear matrix inequality.

\section{Introduction}

By repeating the same operation, a person gradually comes to understand the essential points, and can finally operate with an extremely high precision. This is a process of learning and gradual progress. Repetitive Control (RC) introduces this kind of human learning capability to a control system. From the standpoint of control theory, the self-learning mechanism of an RC System (RCS) involves embedding an internal model of a periodic signal in a repetitive controller (Inoue et al., 1981). As shown in Fig. 11 the repetitive controller, $C_{R}(s)$, contains a puredelay positive-feedback line with a repetition period, $T$. It is used to carry out learning in the following way: the control input, $v(t-T)$, of the previous period is added to the control input, $v(t)$, of the present period to regulate the current control input. This allows the tracking error to be reduced step by step and, finally, the output tracks the periodic reference input without steady-state error.

Linear Repetitive Processes (LRPs) and Iterative Learning Control (ILC) are other two schemes that use the control experience of previous periods for regulation and are closely related to RC. LRPs and ILC are essentially equivalent (Rogers et al., 2007; Wu et al., 2011a). However, as pointed out by Songschon and Longman (2003), Hladowski et al. (2012), Bristow et al. (2006) or She et al. (2012), there are significant differences between them. First, the setting of the initial conditions for each trial is different. RC is intended for continuous operation, whereas ILC (or the LRP) is intended for discontinuous operation. For example, RC might be applied to the control of the reading and writing head of a hard disk drive. Each trial is a full rotation of the disk, and the next trial follows the current one. On the other hand, ILC might be applied to control a robot that performs a task, returns to

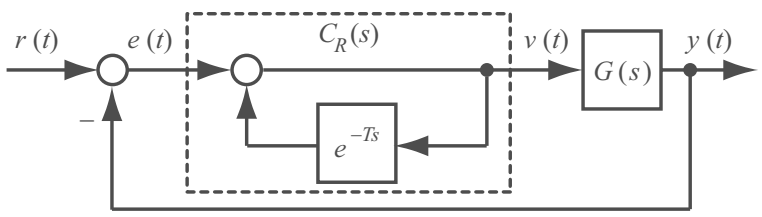

Fig. 1. Configuration of a basic repetitive-control system. 


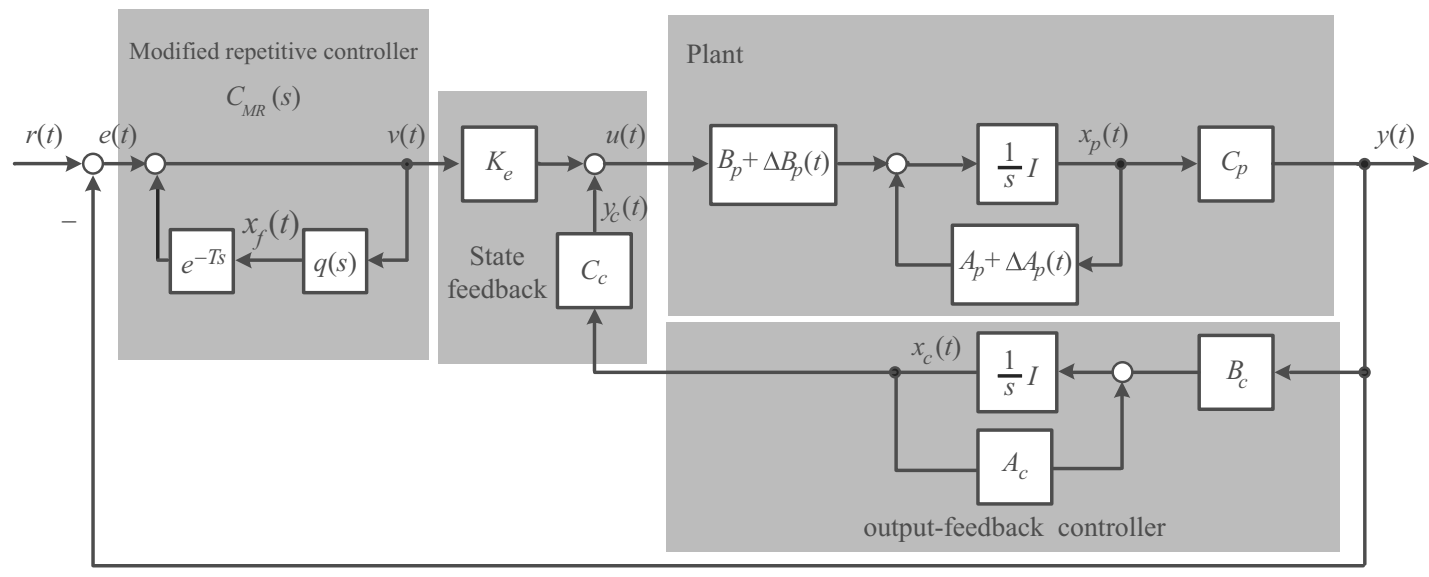

Fig. 2. Configuration of a modified repetitive-control system with a dynamic output-feedback controller.

its home position, and rests until the next task starts.

The difference in the setting of initial conditions leads to different criteria of convergence. For an RCS, we check if the state converges continuously to the steady one. In contrast, since an ILCS always starts from the same state at the beginning of each period, we check if the trial-to-trial error converges; that is, $\left\|e_{i+1}\right\| \leq \lambda\left\|e_{i}\right\|, 0<$ $\lambda<1, i \in\{1,2,3, \cdots\}$, where $i$ is the trial number. Second, the involved stabilization problem is different. In an RCS, learning occurs through periodic delay-based updates in a neutral-type delay system (Fig. 11). The transfer function of this learning mechanism contains an infinite number of poles on the imaginary axis. As a result, an RCS can only be stabilized when the relative degree of the plant is zero (Hara et al., 1988). This restriction does not exist in an ILCS, which is easy to stabilize even for a strictly proper plant. So, the stability conditions for an ILCS or LRPS given by Rogers et al. (2007) and Galkowski et al. (2003), which used Linear Matrix Inequalities (LMIs) and a 2D system approach, cannot be directly extended to handle an RCS.

For a plant with a nonzero relative degree or, in other words, for a strictly proper plant, Hara et al. (1988) devised a Modified Repetitive-Control System (MRCS) by embedding a low-pass filter in the delay line of $C_{R}(s)$. The role of the low-pass filter is to move all of the poles on the imaginary axis to the left half-plane. It relaxes the stability condition at the expense of tracking performance for periodic signals in the high-frequency band. For an MRCS, Li and Yang (2011) proposed a design method for an $H_{\infty}$ robust repetitive controller with output-feedback. It used the output-feedback controller to robustly stabilize the closed-loop system and introduced a feedforward gain into the repetitive controller to improve the dynamic performance and the control accuracy. However, the forward coefficient was determined by trial and error.
RC has an inherent two-dimensional (2D) structural characteristics in that it actually involves two different actions: continuous control within one repetition period and discrete learning between periods. However, most design methods developed for one-dimensional (1D) space (the time domain) ignore the difference between the two actions and only consider their overall effect. That makes it very difficult to dramatically improve the transient performance (see Roncero-Sanchez et al., 2009; Jarzebowska, 2008). In a 2D system setting, Xie and Du (2002), Wu et al. (2008; 2012; 2010; 2011b), Zhou et al. (2012) and She et al. (2012) converted the problem of system design into that of robustly stabilizing a continuousdiscrete 2D system. Unlike 1D methods, they enable the preferential adjustment of control and learning. The resulting control systems exhibit both satisfactory robustness and good tracking performance. However, Wu et al. (2010; 2011b) only considered the case in which the relative degree of the controlled plant is zero (that is, a proper plant); Zhou et al. (2012) and She et al. (2012) extended the result to handle a plant with its relative degree larger than zero (that is, a strictly proper plant). But it requires that the whole state of the plant be available. This may be very hard in many practical applications because of cost and/or difficulty of the installation of sensors.

To enable the method proposed by Wu et al. (2010; 2011b), Zhou et al. (2012), and She et al. (2012) to handle a larger class of systems, this paper presents the configuration of an MRCS with a dynamic output-feedback controller. It deals with the problem of designing a robust MRCS for a class of strictly proper linear plants with time-varying periodic uncertainties that has satisfactory performance in both transient and steady states. First, a continuous-discrete 2D model is established to describe the features of the MRCS. Then, the stability theory of delay systems and the Singular-Value Decomposition (SVD) 
of the output matrix are used to derive a linear-matrixinequality-based robust-stability condition. Two tuning parameters in the LMI manipulate the preferential adjustment of control and learning. Finally, a numerical example demonstrates the validity of the method.

Throughout this paper, $\mathbb{R}^{+}$is the set of non-negative real numbers, $\mathbb{C}^{p}$ is $p$-dimensional vector space over complex numbers; $\mathbb{Z}^{+}$is the set of non-negative integers, $\aleph$ is the linear space of all the functions from $[0, T]$ to $\mathbb{C}^{p}$. $L_{2}\left(\mathbb{R}^{+}, \mathbb{C}^{p}\right)$ is the linear space of square integrable functions from $\mathbb{R}^{+}$to $\mathbb{C}^{p}$, and $\ell_{2}\left(\mathbb{Z}^{+}, \aleph\right)$ is the linear space of all the functions from $\mathbb{Z}^{+}$to $\aleph$, while

$$
\left[\begin{array}{cc}
\Xi & \Upsilon \\
* & \Omega
\end{array}\right]:=\left[\begin{array}{cc}
\Xi & \Upsilon \\
\Upsilon^{T} & \Omega
\end{array}\right]
$$

\section{Problem description}

For the MRCS (Fig. 2), $r(t)$ is a periodic reference input with a period of $T$ and $e(t)=r(t)-y(t)$ is the tracking error. $C_{M R}(s)$ is the modified repetitive controller, and $q(s)$ is a first-order low-pass filter,

$$
q(s)=\frac{\omega_{c}}{s+\omega_{c}},
$$

where $\omega_{c}$ signifies the cutoff angular frequency. Thus, the state-space representation of the modified repetitive controller is

$$
\left\{\begin{array}{l}
\dot{x}_{f}(t)=-\omega_{c} x_{f}(t)+\omega_{c} x_{f}(t-T)+\omega_{c} e(t), \\
v(t)=e(t)+x_{f}(t-T),
\end{array}\right.
$$

where $x_{f}(t)$ is the state variable and $v(t)$ is the output of the modified repetitive controller.

In Fig. 2, the compensated Single-Input, SingleOutput (SISO) uncertain plant is

$$
\left\{\begin{array}{l}
\dot{x}_{p}(t)=\left(A_{p}+\Delta A(t)\right) x_{p}(t)+\left(B_{p}+\Delta B(t)\right) u(t), \\
y(t)=C_{p} x_{p}(t)
\end{array}\right.
$$

where $x_{p}(t) \in \mathbb{R}^{n}$ is the state variable, $u(t), y(t) \in \mathbb{R}$ are the control input and output variables, respectively, while $A_{p}, B_{p}$, and $C_{p}$ are real constant matrices.

Assume that the uncertainties of the plant are

$$
\left[\begin{array}{ll}
\Delta A(t) & \Delta B(t)
\end{array}\right]=M F(t)\left[\begin{array}{ll}
N_{0} & N_{1}
\end{array}\right],
$$

where $M, N_{0}$, and $N_{1}$ are known constant matrices and $F(t)$ is a real, unknown, and time-varying matrix with Lebesgue measurable elements satisfying

$$
F^{T}(t) F(t) \leq I, \quad \forall t>0 .
$$

Assumption 1. The uncertainties, $\Delta A(t)$ and $\Delta B(t)$, vary periodically with the same period as that of the reference input, $r(t)$, i.e., $\forall t>0$,

$$
\Delta A(t+T)=\Delta A(t), \quad \Delta B(t+T)=\Delta B(t) .
$$

Assumption 1 holds in many control engineering problems. For example, the stiffness of a chucked workpiece in a chuck-workpiece system varies periodically and causes parametric vibration in the cutting process (Doi et al., 1985); the dynamics of a helicopter rotor and the horizontal plane motion of ships in waves can be modeled as a periodically time-varying system (Verdult et al., 2007). In addition, a nonlinear system can be approximately represented by a linear periodic model if we linearize it around a steady-state repetitive operating path.

Remark 1. Due to the existence of the periodic uncertainties (4) and (6), the linear plant (3) always generates undesired high harmonics, which are integral multiples of the fundamental frequency, even if the reference signal simply varies sinusoidally (Omata et al., 1985). We can use a repetitive controller to produce these harmonic components. Consequently, a repetitive controller is able to suppress this effect. In this paper, the period of uncertainty is the same as that of the reference input. If these two periods are different rational numbers, we only need to choose the period of the repetitive controller to be their least common multiple so as to guarantee robust tracking.

The following dynamic output-feedback controller is used to stabilize the system:

$$
\left\{\begin{array}{l}
\dot{x}_{c}(t)=A_{c} x_{c}(t)+B_{c} y(t) \\
y_{c}(t)=C_{c} x_{c}(t)
\end{array}\right.
$$

where $A_{c}, B_{c}$, and $C_{c}$ are design parameters to be selected.

Based on the output of the dynamic output-feedback controller and the modified repetitive controller, a linear $\mathrm{RC}$ law is of the form

$$
\begin{aligned}
u(t) & =K_{e} v(t)+y_{c}(t) \\
& =K_{e} v(t)+C_{c} x_{c}(t), \quad K_{e} \in \mathbb{R}
\end{aligned}
$$

where $K_{e}$ is used to enhance the convergence and improve the tracking performance, and $C_{c}$ is used to guarantee the system stability.

Remark 2. Since the tracking accuracy of the modified repetitive-control system depends on the state of the dynamic output-feedback controller, the three undetermined parameters $A_{c}, B_{c}$, and $C_{c}$ in (7) provide design freedom and thus improve the tracking performance of the MRCS. This is an advantage over other methods.

This paper considers the following design problem.

For a given cutoff frequency, $\omega_{c}$, design a dynamic outputfeedback controller (7) and find a suitable control gain, $K_{e}$, in (8) that guarantee the asymptotic stability of the MRCS in Fig. 2

As mentioned in Introduction, $\mathrm{RC}$ involves continuous control and discrete learning. Note that the words 
control and learning mean that we use information on the present and previous periods, respectively, to produce the present control input. Motivated by the fact that a design method that enables preferential adjustment of these two actions can potentially provide better transient performance and better tracking performance, below we present a 2D description for the MRCS in Fig. 2

First, employing the lifting technique (Yamamoto, 1994) to slice the time axis, $[0,+\infty)$, into intervals of length $T$, we convert a vector-valued continuous-time signal, $\xi(t)$, into a function-valued discrete-time sequence, $\xi_{k}(\tau)$. Its element is denoted by $\xi(k, \tau)$ in this paper. That is, we have

$$
\begin{aligned}
& \xi(k, \tau)=\xi_{k}(\tau):=\mathcal{L}_{\mathcal{C}}[\xi(t)], \\
& t=k T+\tau, \quad \tau \in[0, T], \quad k \in \mathbb{Z}^{+},
\end{aligned}
$$

where $\mathcal{L}_{\mathcal{C}}$ is an isometric and isomorphic transformation between $L_{2}\left(\mathbb{R}^{+}, \mathbb{C}^{p}\right)$ and $\ell_{2}\left(\mathbb{Z}^{+}, \aleph\right)$. Setting $r(t)=0$ thus yields the following $2 \mathrm{D}$ representation from (2), (3), (7), and (8):

$$
\begin{aligned}
& \left\{\begin{array}{l}
\dot{x}(k, \tau)=A x(k, \tau)+A_{1} x(k-1, \tau)+B u(k, \tau), \\
v(k, \tau)=C x(k, \tau)+C_{1} x(k-1, \tau),
\end{array}\right. \\
& u(k, \tau)=F_{p} x(k, \tau)+F_{e} x(k-1, \tau),
\end{aligned}
$$

where

$$
\begin{aligned}
& x^{T}(k, \tau)=\left[x_{p}^{T}(k, \tau) x_{f}^{T}(k, \tau) x_{c}^{T}(k, \tau)\right], \\
& A=\left[\begin{array}{ccc}
A_{p}+\Delta A(k, \tau) & 0 & 0 \\
-\omega_{c} C_{p} & -\omega_{c} & 0 \\
B_{c} C_{p} & 0 & A_{c}
\end{array}\right], \\
& A_{1}=\left[\begin{array}{ccc}
0 & 0 & 0 \\
0 & \omega_{c} & 0 \\
0 & 0 & 0
\end{array}\right], B=\left[\begin{array}{c}
B_{p}+\Delta B(k, \tau) \\
0 \\
0
\end{array}\right], \\
& C=\left[\begin{array}{lll}
-C_{p} & 0 & 0
\end{array}\right], C_{1}=\left[\begin{array}{lll}
0 & 1 & 0
\end{array}\right] \text {, } \\
& F_{p}=\left[\begin{array}{lll}
F_{p 1} & 0 & F_{p 3}
\end{array}\right], \quad F_{e}=\left[\begin{array}{lll}
0 & F_{e 2} & 0
\end{array}\right], \\
& F_{p 1}=-K_{e} C_{p}, \quad F_{p 3}=C_{c}, \quad F_{e 2}=K_{e} \text {. }
\end{aligned}
$$

The above continuous-discrete 2D model converts the design problem into the robust stabilization of the continuous-discrete 2D system (10) under the control law (11). Note that the 2D control law (11) contains the direct sum of the effects of control and learning. This allows us to adjust control and learning preferentially by changing $F_{p}$ and $F_{e}$, which is the big advantage over 1D methods. In addition, from (13), the control gains in (8) can be rewritten as

$$
K_{e}=-F_{p 1} C_{p}^{T} \hat{C}^{-1},
$$

where $\hat{C}=C_{p} C_{p}^{T}$.
Substituting the control input 110 into the system (10) yields the closed-loop MRCS:

$$
\left\{\begin{array}{r}
\dot{x}(k, \tau)=A_{l} x(k, \tau)+A_{d l} x(k-1, \tau) \\
+M_{l} \Gamma(k, \tau)+M_{l} \tilde{\Gamma}(k, \tau), \\
v(k, \tau)=C x(k, \tau)+C_{1} x(k-1, \tau),
\end{array}\right.
$$

where

$$
\begin{aligned}
A_{l} & =\left[\begin{array}{ccc}
A_{p}+B_{p} F_{p 1} & 0 & B_{p} F_{p 3} \\
-\omega_{c} C_{p} & -\omega_{c} & 0 \\
B_{c} C_{p} & 0 & A_{c}
\end{array}\right], \\
A_{d l} & =\left[\begin{array}{ccc}
0 & B_{p} F_{e 2} & 0 \\
0 & \omega_{c} & 0 \\
0 & 0 & 0
\end{array}\right], \quad M_{l}=\left[\begin{array}{c}
M \\
0 \\
0
\end{array}\right], \\
\Gamma(k, \tau) & =F(k, \tau) \Psi x(k, \tau), \\
\tilde{\Gamma}(k, \tau) & =F(k, \tau) \tilde{\Psi} x(k-1, \tau), \\
\Psi & =\left[\begin{array}{lll}
N_{0}+N_{1} F_{p 1} & 0 & N_{1} F_{p 3}
\end{array}\right], \\
\tilde{\Psi} & =\left[\begin{array}{lll}
0 & N_{1} F_{e 2} & 0
\end{array}\right] .
\end{aligned}
$$

Equation (15) shows that there is a coupling relationship between control and learning, and they affect each other by means of $A_{l}$ and $A_{d l}$.

\section{Design of a robust MRCS}

Definition 1. (Zhou et al., 1996) Assume that the output matrix $C_{p} \in \mathbb{R}^{m \times n}$ in (3) has full row rank. The SVD of $C_{p}$ is

$$
C_{p}=U\left[\begin{array}{ll}
S & 0
\end{array}\right] V^{T} .
$$

where $S \in \mathbb{R}^{m \times m}$ is a diagonal matrix with positive, diagonal elements in decreasing order, $0 \in \mathbb{R}^{m \times(n-m)}$ is a zero matrix, $U \in \mathbb{R}^{m \times m}$ and $V \in \mathbb{R}^{n \times n}$ are unitary matrices.

The following lemma presents an equivalent condition for the matrix equation $C_{p} X=\bar{X} C_{p}$.

Lemma 1. (Ho and Lu, 2003) For a given $C_{p} \in \mathbb{R}^{m \times n}$ with $\operatorname{rank}\left(C_{p}\right)=m$, if $X \in \mathbb{R}^{n \times n}$ is a symmetric matrix, then there exists a matrix, $\bar{X} \in \mathbb{R}^{m \times m}$, such that $C_{p} X=$ $\bar{X} C_{p}$ holds if and only if

$$
X=V \operatorname{diag}\left\{X_{11}, X_{22}\right\} V^{T},
$$

where $X_{11} \in \mathbb{R}^{m \times m}$ and $X_{22} \in \mathbb{R}^{(n-m) \times(n-m)}$.

Lemma 2. (Schur complement (cf. Khargonek et al., 1990)) For any real matrix $\Sigma=\Sigma^{T}$, the following assertions are equivalent:
1. $\Sigma=\left[\begin{array}{cc}S_{11} & S_{12} \\ * & S_{22}\end{array}\right]<0$,
2. $S_{11}<0$ and $S_{22}-S_{12}^{T} S_{11}^{-1} S_{12}<0$, and
3. $S_{22}<0$ and $S_{11}-S_{12} S_{22}^{-1} S_{12}^{T}<0$. 
Lemma 3. (Petersen and Hollot, 1986) For given matrices $\Upsilon=\Upsilon^{T}, H$, and $E$ with appropriate dimensions,

$$
\Upsilon+H F(t) E+E^{T} F^{T}(t) H^{T}<0
$$

holds for all $F(t)$ satisfying $F^{T}(t) F(t) \leq I$ if and only if there exists an $\varepsilon>0$ such that

$$
\Upsilon+\varepsilon H H^{T}+\varepsilon^{-1} E^{T} E<0 .
$$

An RC process is basically continuous. Accordingly, for any variable $\xi(t) \in \mathbb{C}^{p}$ of the MRCS in Fig. 2 the image of lifting (10), $\xi(k, \tau)$, satisfies the following boundary condition:

$$
\xi(k+1,0)=\xi(k, T), \quad k \in \mathbb{Z}^{+} .
$$

Note that 17 provides the fact that, if $\xi(k, \tau)$ decreases monotonically with $\tau$ in $[0, T]$ for $k \in \mathbb{Z}^{+}$, then $\xi(k, \tau)$ decreases monotonically with $k$ for any fixed $\tau \in[0, T]$. Thus, $\xi(t)$ decreases monotonically in the interval $[0,+$ $\infty)$. So, the dynamic-output-feedback based MRCS in Fig. 2 is asymptotically stable if and only if there exists a semi-positive definite functional $V(k, \tau)$ that is continuous and decreases monotonically in every interval $[k T,(k+1) T], k \in\{0,1,2,3, \ldots\}$ (She et al., 2012).

Based on the above definition and lemmas, we obtain the following theorem.

Theorem 1. For a given cutoff angular frequency, $\omega_{c}$, and two positive scalars, $\alpha$ and $\beta$, if there exist symmetrical and positive-definite matrices $X_{11}, X_{22}, X_{2}, X_{3}, Y_{1}$, $Y_{2}$, and $Y_{3}$, and arbitrary matrices $W_{1}, W_{2}, W_{3}, W_{4}$, and $W_{5}$ such that

$$
\left[\begin{array}{cc}
\Theta & \Pi \\
\Pi^{T} & \Xi
\end{array}\right]<0
$$

where

$$
\begin{aligned}
\Theta & =\left[\begin{array}{cccccccc}
\Theta_{11} & \Theta_{12} & \Theta_{13} & 0 & \Theta_{15} & 0 & \varepsilon M & \varepsilon M \\
* & \Theta_{22} & 0 & 0 & \Theta_{25} & 0 & 0 & 0 \\
* & * & \Theta_{33} & 0 & 0 & 0 & 0 & 0 \\
* & * & * & -Y_{1} & 0 & 0 & 0 & 0 \\
* & * & * & * & -\beta Y_{2} & 0 & 0 & 0 \\
* & * & * & * & * & -Y_{3} & 0 & 0 \\
* & * & * & * & * & * & -\varepsilon I & 0 \\
* & * & * & * & * & * & * & -\varepsilon I
\end{array}\right], \\
\Pi & =\left[\begin{array}{ccccccc}
X_{1} & 0 & 0 & X_{1} N_{0}^{T}+W_{1}^{T} N_{1}^{T} & 0 \\
0 & X_{2} & 0 & 0 & 0 \\
0 & 0 & \alpha X_{3} & \alpha W_{2}^{T} N_{1}^{T} & 0 \\
0 & 0 & 0 & 0 & 0 \\
0 & 0 & 0 & 0 & \beta W_{4}^{T} N_{1}^{T} \\
0 & 0 & 0 & 0 & 0 \\
0 & 0 & 0 & 0 & 0 \\
0 & 0 & 0 & 0 & 0
\end{array}\right], \\
\Xi & =\operatorname{diag}\left\{-Y_{1}-\beta Y_{2}-Y_{3}-\varepsilon I-\varepsilon I\right\}, \\
\Theta_{11} & =A_{p} X_{1}+X_{1} A_{p}^{T}+B_{p} W_{1}+W_{1}^{T} B_{p}^{T},
\end{aligned}
$$

$$
\begin{aligned}
\Theta_{15} & =\beta B_{p} W_{4}, \quad \Theta_{22}=-2 \omega_{c} X_{2}, \\
\Theta_{25} & =\beta \omega_{c} Y_{2}, \quad \Theta_{33}=\alpha W_{5}+\alpha W_{5}^{T}, \\
X_{1} & =V \operatorname{diag}\left\{X_{11}, X_{22}\right\} V^{T},
\end{aligned}
$$

then the MRCS (15) in Fig. 2 is asymptotically stable. Furthermore, the parameters of the dynamic outputfeedback controller (7) are

$$
\begin{aligned}
& A_{c}=W_{5} X_{3}^{-1}, \\
& B_{c}=W_{3} U S X_{11}^{-1} S^{-1} U^{T}, \\
& C_{c}=W_{2} X_{3}^{-1}
\end{aligned}
$$

where $U$ and $V$ are unitary matrices defined in SVD (16), and the $2 D$ control gains in (12) are

$$
\begin{aligned}
& F_{p 1}=W_{1} X_{1}^{-1} \\
& F_{p 3}=W_{2} X_{3}^{-1}, \\
& F_{e 2}=W_{4} Y_{2}^{-1} .
\end{aligned}
$$

Proof. Let

$$
\begin{aligned}
& P_{1}=X_{1}^{-1}, \quad P_{2}=X_{2}^{-1}, \quad P_{3}=X_{3}^{-1}, \\
& Q_{1}=Y_{1}^{-1}, \quad Q_{2}=Y_{2}^{-1}, \quad Q_{3}=Y_{3}^{-1} \text {, }
\end{aligned}
$$

and choose a Lyapunov functional candidate to be

$$
\begin{aligned}
V(k, \tau) & =V_{1}(k, \tau)+V_{2}(k, \tau) \\
V_{1}(k, \tau) & =x^{T}(k, \tau) \operatorname{Px}(k, \tau) \\
V_{2}(k, \tau) & =\int_{\tau-T}^{\tau} x^{T}(k, s) Q x(k, s) \mathrm{d} s
\end{aligned}
$$

where

$$
P=\operatorname{diag}\left\{P_{1}, P_{2}, \frac{1}{\alpha} P_{3}\right\}, \quad Q=\operatorname{diag}\left\{Q_{1}, \frac{1}{\beta} Q_{2}, Q_{3}\right\} .
$$

Here, $V_{1}(k, \tau)$ and $V_{2}(k, \tau)$ are two quadratic terms that are directly related to the control and learning performance, respectively, while $P$ and $Q$ can be any positive definite matrices. This is a standard choice in an $n \mathrm{D}$ system, and a simple check shows that $V(k, \tau)>0$ for any $x(k, \tau) \neq 0$ and $V(k, \tau)=0$ when $x(k, \tau)=0$. In order to carry out the preferential adjustment of control and learning, we choose $P$ and $Q$ as block diagonal matrices.

Based on the above analysis, we only need to consider the associated increment of $V(k, \tau)$ in every interval $[k T,(k+1) T], k \in\{0,1,2,3, \ldots\}$. Along the time trajectory of (15), we have

$$
\frac{\mathrm{d} V(k, \tau)}{\mathrm{d} \tau}=\eta^{T}(k, \tau) \Lambda \eta(k, \tau)
$$


where

$$
\begin{aligned}
& \eta(k, \tau)=\left[\begin{array}{ll}
x^{T}(k, \tau) & x^{T}(k-1, \tau)
\end{array}\right]^{T}, \\
& \Lambda=\Upsilon+H F(k, \tau) E+E^{T} F^{T}(k, \tau) H^{T} \\
& +H F(k, \tau) \bar{E}+\bar{E}^{T} F^{T}(k, \tau) H^{T}, \\
& \Upsilon=\left[\begin{array}{cc}
P A_{l}+A_{l}^{T} P+Q & P A_{d l} \\
* & -Q
\end{array}\right], \\
& H^{T}=\left[\begin{array}{cccccc}
M^{T} P_{1}^{T} & 0 & 0 & 0 & 0 & 0
\end{array}\right] \text {, } \\
& E=\left[\begin{array}{llll}
\Psi & 0 & 0 & 0
\end{array}\right] \text {, } \\
& \bar{E}=\left[\begin{array}{llll}
0 & \bar{\Psi} & 0 & 0
\end{array}\right],
\end{aligned}
$$

and $A_{l}, A_{d l}, \Psi$, and $\bar{\Psi}$ are defined in (15).

Clearly, if

$$
\begin{aligned}
\Upsilon & +H F(k, \tau) E+E^{T} F^{T}(k, \tau) H^{T} \\
& +H F(k, \tau) \bar{E}+\bar{E}^{T} F^{T}(k, \tau) H^{T}<0,
\end{aligned}
$$

then

$$
\frac{\mathrm{d} V(k, \tau)}{\mathrm{d} \tau}<0 .
$$

Applying Lemma 3 to 26 shows that the inequality (26) holds if and only if there exists an $\varepsilon>0$ such that

$$
\Upsilon+2 \varepsilon H H^{T}+\frac{1}{\varepsilon} E^{T} E+\frac{1}{\varepsilon} \bar{E}^{T} \bar{E}<0 .
$$

Also, from Lemma 2, 28) is equivalent to the following matrix inequality:

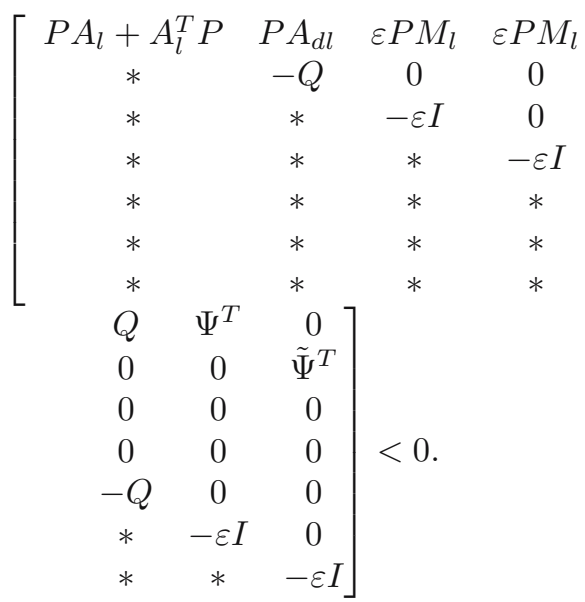

Since $X_{1}=V \operatorname{diag}\left\{X_{11}, X_{22}\right\} V^{T}$, there exists a matrix, $\bar{X}_{1}$, such that

$$
C_{p} X_{1}=\bar{X}_{1} C_{p} .
$$

Also, we have

$$
\begin{aligned}
\bar{X}_{1} & =U S X_{11} S^{-1} U^{T}, \\
\bar{X}_{1}^{-1} & =U S X_{11}^{-1} S^{-1} U^{T} .
\end{aligned}
$$

Define

$\left\{\begin{array}{l}W_{1}=F_{p 1} X_{1}, \quad W_{2}=F_{p 3} X_{3}, \quad W_{3}=B_{c} \bar{X}_{1}, \\ W_{4}=F_{e 2} Y_{2}, \quad W_{5}=A_{c} X_{3} .\end{array}\right.$
Pre- and post-multiplying the matrix on the left side of (29) by

$$
\operatorname{diag}\left\{X_{1}, X_{2}, \alpha X_{3}, Y_{1}, \beta Y_{2}, Y_{3}, I, I, Y_{1}, \beta Y_{2}, Y_{3}, I, I\right\}
$$

and substituting (30) and (32) into (29) yield the required LMI of (18). The parameters of the dynamic outputfeedback controller in 20, and control law matrices in (21) can be calculated from (32).

Theorem 1 constituted an LMI-based design method for the MRCS in Fig. 2. As for the cutoff frequency, $\omega_{c}$, of the low-pass filter $q(s)$ in (1), it can be determined by the system operating frequency range. Combining the technique of matrix-linear-decomposition given in the work of She et al. (2012) and Theorem 1, it is easy to develop an iterative algorithm to optimize both the maximum cutoff angular frequency and the parameters of the stabilization controller.

Remark 3. Two tuning parameters $\alpha$ and $\beta$ in the LMI (18) are used to justify the choice of 2D control gains, $F_{p}$ and $F_{e}$, and thereby enable the preferential adjustment of control and learning. More specifically, we use $\alpha$ to adjust the weight matrix $P_{3}$ and $\beta$ to adjust the weight matrix $Q_{2}$, and thus regulate the corresponding feasible solutions $F_{p 3}$ and $F_{e 2}$.

From Theorem 1 we can directly obtain a sufficient stability condition for the nominal continuous-discrete 2D closed-loop system

$$
\left\{\begin{array}{l}
\dot{x}(k, \tau)=A_{l} x(k, \tau)+A_{d l} x(k-1, \tau), \\
v(k, \tau)=C x(k, \tau)+C_{1} x(k-1, \tau),
\end{array}\right.
$$

where $A_{l}, A_{d l}, C$, and $C_{1}$ are defined in (15).

Corollary 1. For a given cutoff angular frequency, $\omega_{c}$, and two positive scalars, $\alpha$ and $\beta$, if there exist symmetrical and positive-definite matrices $X_{11}, X_{22}, X_{2}, X_{3}, Y_{1}$, $Y_{2}$, and $Y_{3}$, and arbitrary matrices $W_{1}, W_{2}, W_{3}, W_{4}$, and $W_{5}$ such that the LMI

$$
\left[\begin{array}{cccccc}
\Theta_{11} & \Theta_{12} & \Theta_{13} & 0 & \Theta_{15} & 0 \\
* & \Theta_{22} & 0 & 0 & \Theta_{25} & 0 \\
* & * & \Theta_{33} & 0 & 0 & 0 \\
* & * & * & -Y_{1} & 0 & 0 \\
* & * & * & * & -\beta Y_{2} & 0 \\
* & * & * & * & * & -Y_{3} \\
* & * & * & * & * & * \\
* & * & * & * & * & * \\
* & * & * & * & * & * \\
X_{1} & 0 & 0 \\
0 & & X_{2} & 0 \\
0 & 0 & \alpha X_{3} \\
0 & 0 & 0 & & \\
0 & & 0 & 0 & \\
0 & 0 & 0 \\
-Y_{1} & 0 & 0 \\
* & -\beta Y_{2} & 0 \\
* & & & & \\
* & & &
\end{array}\right.
$$


holds, where $X_{1}, \Theta_{11}, \Theta_{12}, \Theta_{13}, \Theta_{15}, \Theta_{22}, \Theta_{25}$, and $\Theta_{33}$ are defined in (18), then the closed-loop system (33) is asymptotically stable. Furthermore, the parameters in (7) and control gains in (13) can be calculated from (20) and (21), respectively.

\section{Numerical example}

In this section, we apply our method to the speed control of a rotational system that consists of two motors: one is the controlled object, and the other is a disturbance generator (Fig. 3. (cf., She et al., 2008)). Their axles are coupled together with a spring. The state-space model matrices of the uncertain plant were identified to be

$$
\left\{\begin{aligned}
A_{p}= & {\left[\begin{array}{ccc}
-31.31 & 0 & -2.833 \times 10^{4} \\
0 & -10.25 & 8001 \\
1 & -1 & 0
\end{array}\right], } \\
B_{p}= & {\left[\begin{array}{c}
28.06 \\
0 \\
0
\end{array}\right], \quad C_{p}=\left[\begin{array}{lll}
1 & 0 & 0
\end{array}\right], } \\
M= & {\left[\begin{array}{lll}
1 & 0 & 0 \\
0 & 1 & 0 \\
0 & 0 & 1
\end{array}\right], \quad N_{0}=\left[\begin{array}{lll}
0 & 0 & 0.1 \\
0 & 0 & 0.01 \\
0 & 0 & 0
\end{array}\right], } \\
N_{1}= & {\left[\begin{array}{l}
0 \\
0 \\
0
\end{array}\right], F(t)=\left[\begin{array}{ccc}
\sin \pi t & 0 & 0 \\
0 & \sin \pi t & 0 \\
0 & 0 & \sin \pi t
\end{array}\right] . }
\end{aligned}\right.
$$

We consider the problem of tracking the following periodic reference input:

$$
r(t)=\sin \pi t+0.5 \sin 2 \pi t+0.5 \sin 3 \pi t .
$$

The repetition period is

$$
T=2 \mathrm{~s} .
$$

Let the cutoff frequency be

$$
\omega_{c}=200 \mathrm{rad} / \mathrm{s} \text {. }
$$

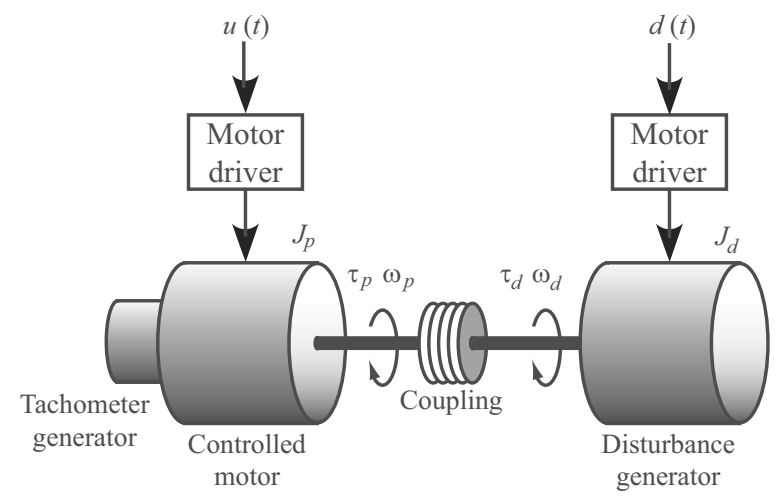

Fig. 3. Rotational control system.

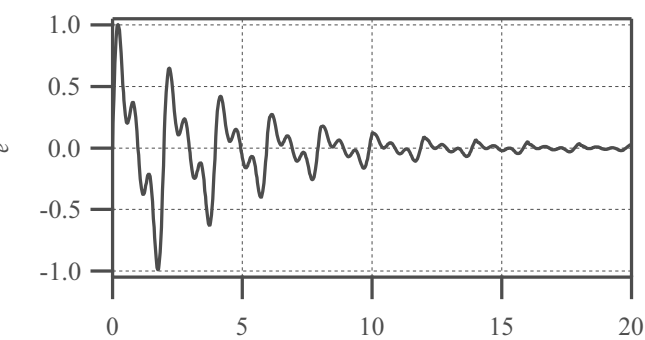

(a) ${ }^{t[\mathrm{~s}]}$

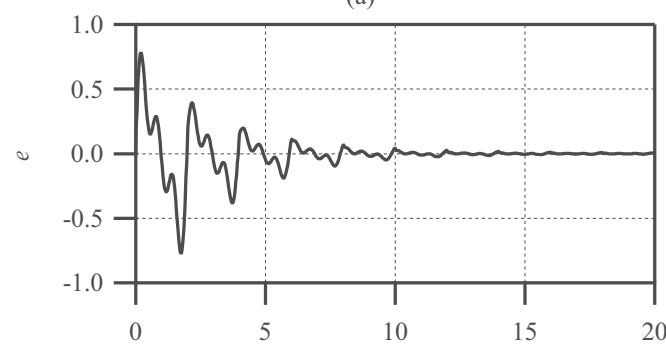

(b) ${ }^{t[\mathrm{~s}]}$

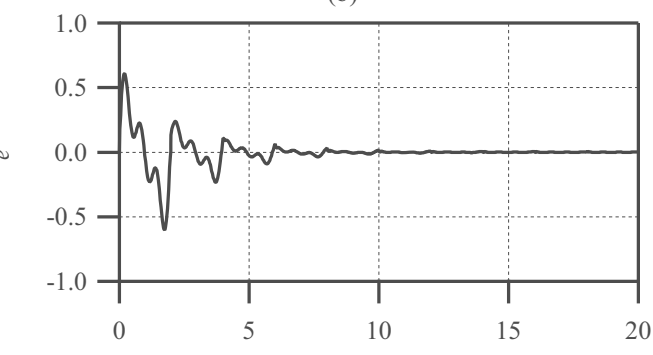

(c) ${ }^{t[\mathrm{~s}]}$

Fig. 4. Tracking error for the parameter sets 40): $\alpha=$ $0.1, \beta=0.01$ (a), $\alpha=0.1, \beta=0.43$ (b), $\alpha=$ $0.8, \beta=0.43$ (c).

Choose the performance index

$$
J_{10}=\frac{1}{2} \sum_{k=0}^{9} \int_{k T}^{(k+1) T} e^{2}(t) \mathrm{d} t
$$

to evaluate the system overall performance and use it as a criterion for the selection of the tuning parameters, $\alpha$ and $\beta$.

To investigate what effect adjusting the tuning parameters has, we carry out simulation for three parameter sets:

$$
\begin{cases}\text { (a) } \quad \alpha=0.1, & \beta=0.01 \\ \text { (b) } \alpha=0.1, & \beta=0.43, \\ \text { (c) } \alpha=0.8, & \beta=0.43 .\end{cases}
$$

The performance indices are

$$
\begin{aligned}
& J_{10}^{a}=0.4984, \\
& J_{10}^{b}=0.2366, \\
& J_{10}^{c}=0.1271 .
\end{aligned}
$$


In the tuning process, the tracking error in the first period shows the control performance, and the convergence speed of the tracking error characterizes the learning efficiency. Figure 4 shows that the tracking error converges faster for set (b) than for (a). Accordingly, adjusting $\beta$ primarily affects learning. Increasing $\alpha$ from 0.1 to 0.8 , set (c) greatly improves the control performance. So, adjusting $\alpha$ primarily affects control. Note that, due to the coupling between control and learning, changing $\alpha$ strongly influences learning, and tuning $\beta$ also influences the control action. This can be observed from the tracking error in Fig. 4 Among the three parameter sets, set (c) provides the best performance in both the transient and steady states.

Employing the following optimization:

$$
\min J_{10} \text { such that (18) holds }
$$

and combining a fixed-step method in the ranges

$$
\alpha \in(0,1], \beta \in(0,1]
$$

yield the best tuning parameter set:

$$
\alpha=0.8, \quad \beta=0.25 \text {. }
$$

The corresponding output-feedback controller parameters are

$$
\left\{\begin{array}{l}
A_{c}=\operatorname{diag}\{-1.3144,-1.3144,-1.3144\}, \\
B_{c}=\left[\begin{array}{lll}
3.8321 & 0 & 0
\end{array}\right]^{T}, C_{c}=\left[\begin{array}{lll}
0.5463 & 0 & 0
\end{array}\right],
\end{array}\right.
$$

and the feed-forward control gain in Fig. 2 is

$$
K_{e}=7.8382 \text {. }
$$

Simulation results in Fig. 5 show that the system is robustly stable for the periodic uncertainties and it enters into the steady state in the fourth period. Moreover, $J_{10}=0.0441$. Compared with the approach of Li and Yang (2011), we only need to change two tuning parameters to improve the convergence speed and the tracking performance. In comparison with the method of Hladowski et al. (2012), who considered the application of discrete ILC to the nominal plant (35), we improve greatly the transient performance through the preferential adjustment of control and learning. In addition, we extend the state-feedback to dynamic output-feedback, which enables RC to be applied to a wider range of control engineering problems.

Furthermore, to verify the robustness of the resulting system, we simulated adding white noise with a Signalto-Noise Ratio (SNR) of $35 \mathrm{~dB}$ to the output. The index

$$
J_{e}=\sup _{t \geq t_{s}}|e(t)|
$$

is used to evaluate the steady-state tracking performance, where $t_{s}$ is the setting time of the control system.
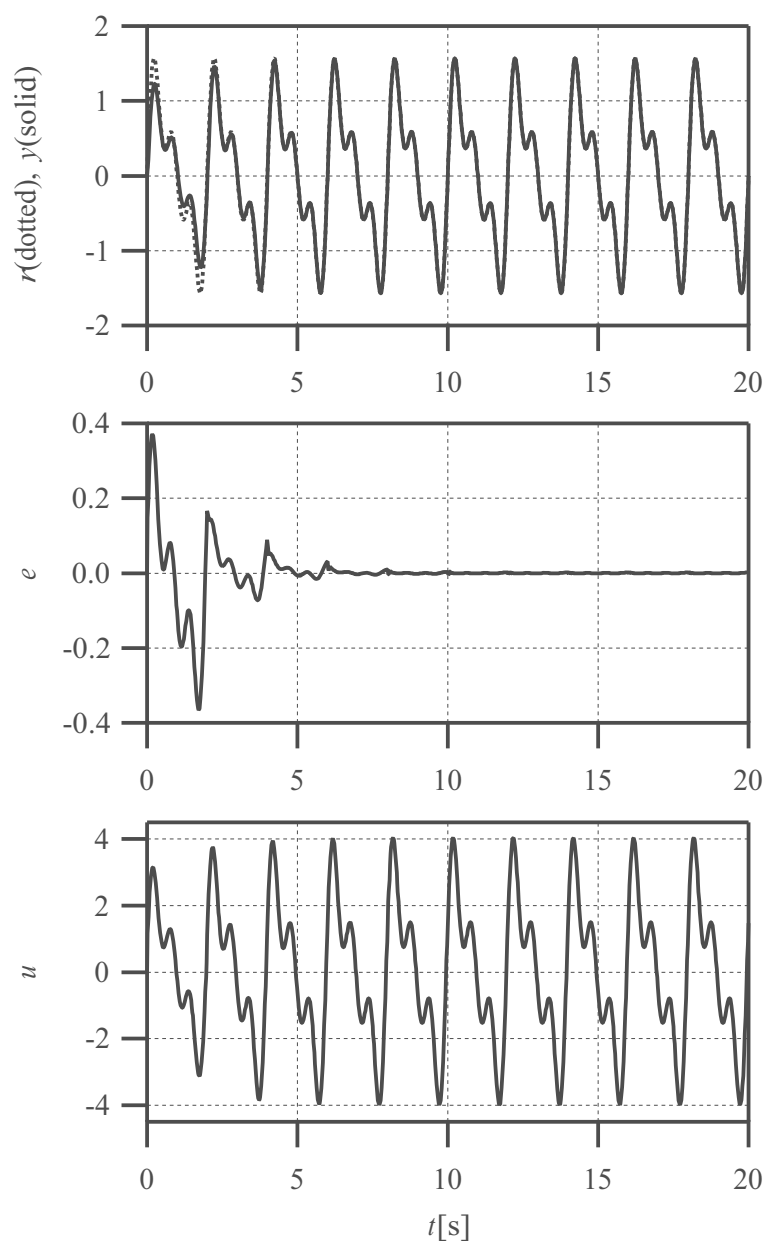

Fig. 5. Simulation results for a robust MRCS in Fig. 2 for $\alpha=$ 0.8 and $\beta=0.25$.

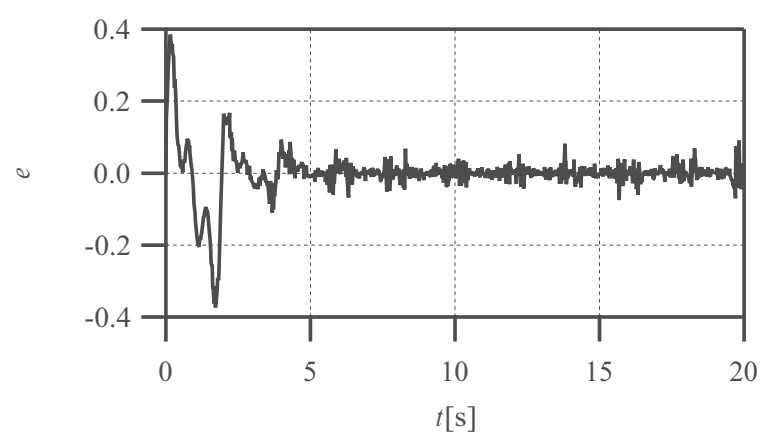

Fig. 6. Steady-state tracking error with white Gaussian noise (SNR: $35 \mathrm{~dB}$ ) in the output.

Using the parameter set in (44), we found that $J_{e}=$ 0.0008 without noise, and $J_{e_{\text {noise }}}=0.0817$ with noise. The simulation results (Fig. 6) show that the control system remains stable and the steady-state tracking error remains very small even with noise. 


\section{Conclusion}

This paper developed an LMI-based method of designing a robust MRCS with a dynamic output-feedback controller for a class of strictly proper plants. A continuousdiscrete 2D model was established that allows preferential adjustment of control and learning by means of the gains in the 2D control law. The combination of Lyapunov stability theory of delay-time systems and the SVD of the output matrix was used to derive an LMI-based sufficient stability condition, and the control gains were given in the form of the feasible solutions of the LMI. Two tuning parameters in the condition enabled the preferential adjustment of control and learning. Finally, simulation results on a two-motor rational system showed that the resulting system has both a satisfactory convergence rate and good tracking performance.

On the other hand, a mechatronic system usually does not have unstable zeros, and we only considered this case in this study. However, it is of theoretical significance to develop a method of designing a robust repetitivecontrol system based on dynamic output-feedback for a plant with unstable zeros. We plan to study this topic in the near future.

\section{Acknowledgment}

This work was supported in part by the National Natural Science Foundation of China (Nos. 61203010, 61210011, and 51374107), Hunan Natural Science Foundation (Grant No. 11JJ4059), and the Scientific Research Fund of Hunan Provincial Education Department (Grant No. 12B044).

\section{References}

Bristow, D.A., Tharayil, M. and Alleyne, A.G. (2006). A survey of iterative learning control, IEEE Control Systems Magazine 26(3): 96-114.

Doi, M., Masuko, M., Ito, Y. and Tezuka, A. (1985). A study on parametric-vibration in chuck work, Bulletin of the Japan Society of Mechanical Engineers 28(245): 2774-2780.

Galkowski, K., Paszke, W., Rogers, E., Xu, S., Lam, J. and Owens, D.H. (2003). Stability and control of differential linear repetitive processes using an LMI setting, IEEE Transactions on Circuits and Systems II: Analog and Digital Signal Processing 50(9): 662-666.

Hara, S., Yamamoto, Y. and Omata, T. (1988). Repetitive control system: A new type servo system for periodic exogenous signals, IEEE Transactions on Automatic Control 33(7): 659-668.

Hladowski, L., Galkowski, K., Rogers, E., Zhou, L., He, Y. and Kummert, A. (2012). Repetitive process based iterative learning control for a two motors system, IEEE International Conference on Control Applications, CCA 2012, Dubrovnik, Croatia, pp. 154-159.
Ho, D.W.C. and Lu, G. (2003). Robust stabilization for a class of discrete-time non-linear system via output feedback: The unified LMI approach, International Journal of Control 76(2): 105-115.

Inoue, T., Nakano, M. and Iwai, S. (1981). High accuracy control of a proton synchrotron magnet power supply, Proceedings of the 8th IFAC World Congress, Kyoto, Japan, pp. 216221.

Jarzebowska, E.M. (2008). Advanced programmed motion tracking control of nonholonomic mechanical systems, IEEE Transactions on Robotics 24(6): 1315-1328.

Khargonek, P.P., Petersen, I.R. and Zhou, K. (1990). Robust stabilization of uncertain linear systems: Quadratic stability and $\mathrm{H}_{\infty}$ control theory, IEEE Transactions on Automatic Control 35(3): 356-361.

Li, Z.D. and Yang, W.D. (2011). $\mathrm{H}_{\infty}$ robust repetitive control with output feedback for roll eccentricity compensation, Control Theory and Applications 28(3): 381-388.

Omata, T., Hara, T. and Nakano, M. (1985). Repetitive control for linear periodic systems, Electrical Engineering in Japan 105(3): 131-138.

Petersen, I.R. and Hollot, C.V. (1986). A Riccati equation approach to the stabilization of uncertain linear systems, $\mathrm{Au}$ tomatica 22(4): 397-411.

Rogers, E., Galkowski, K. and Owens, D.H. (2007). Control Systems Theory and Applications for Linear Repetitive Processes, Lecture Notes in Control and Information Sciences, Vol. 349, Springer-Verlag, Berlin.

Roncero-Sanchez, P., Acha, E. and Ortega-Calderon, J.E. (2009). A versatile control scheme for a dynamic voltage restorer for power-quality improvement, IEEE Transactions on Power Delivery 24(1): 277-284.

She, J., Fang, M. and Ohyama, Y. (2008). Improving disturbance-rejection performance based on an equivalentinput-disturbance approach, IEEE Transactions on Industrial Electronics 55(1): 380-389.

She, J., Zhou, L. and Wu, M. (2012). Design of a modified repetitive-control system based on a continuous-discrete 2D model, Automatica 48(5): 844-850.

Songschon, S. and Longman, R.W. (2003). Comparison of the stability boundary and the frequency response stability condition in learning and repetitive control, International Journal of Applied Mathematics and Computer Science 13(2): 169-177.

Verdult, V., Lovera, M. and Verhaegen, M. (2007). Identification of linear parameter-varying state space models with application to helicopter rotor, International Journal of Control 77(13): 1149-1159.

Wu, L.G., Shi, P., Gao, H.J. and Wang, C.H. (2008). $\mathrm{H}_{\infty}$ filtering for 2D Markovian jump systems, Electric Machines and Control 44(7): 1849-1858.

Wu, M., Zhou, L., She, J. and He, Y. (2010). Design of robust output-feedback repetitive controller for class of linear systems with uncertainties, Science China: Information Sciences 53(5): 1006-1015. 
Wu, L.G., Gao, H.J. and Wang, C.H. (2011). Quasi sliding mode control of differential linear repetitive process with unknown input disturbance, IEEE Transactions on Industrial Electronics (7): 3059-3068.

Wu, M., Zhou, L. and She, J. (2011b). Design of observer-based $\mathrm{H}_{\infty}$ robust repetitive-control system, IEEE Transactions on Automatic Control 56(6): 1452-1457.

Wu, L.G., Yao, X.M. and Zheng, W. X. (2012). Generalized $\mathrm{H}_{2}$ fault detection for Markovian jumping two-dimensional systems, Automatica 48(8): 1741-1750.

Xie, L.H. and Du, C.L. (2002). $H_{\infty}$ Control and Filter of TwoDimensional System, Springer, Berlin.

Yamamoto, Y. (1994). A function space approach to sampled data control systems and tracking problems, IEEE Transactions on Automatic Control 39(4): 703-713.

Zhou, K., Doyle, J.C. and Glover, K. (1996). Robust and Optimal Control, Prentice Hall, Upper Saddle River, NJ.

Zhou, L., She, J. and Wu, M. (2012). Design of a robust modified repetitive-control system for a periodic plant, ASME Journal of Dynamic Systems, Measurement, and Control 134(1): 011023-1-7.

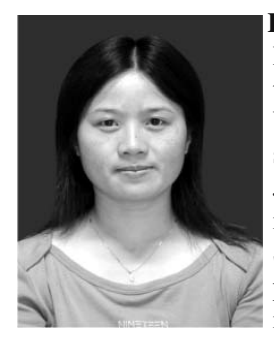

Lan Zhou received the B.Sc. degree in 1998 from Hunan Normal University, Changsha, China, and the M.Sc. degree in 2006 from Central South University, Changsha. From 2008 to 2010, she was a joint cultivation doctoral candidate of Japan and China. She received her Ph.D. degree in control science and engineering from Central South University in 2011. She is an associate professor of control theory and control engineering with the School of Information and Electrical Engineering, Hunan University of Science and Technology, Xiangtan, China. Her current research interests include robust control, repetitive control, and control applications.

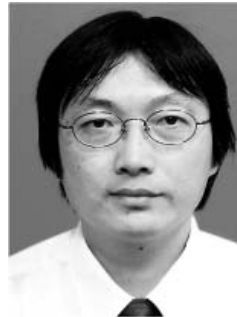

Jinhua She received a B.Sc. in engineering from Central South University, Changsha, Hunan, China, in 1983, and an M.Sc. (1990) and a Ph.D. (1993) in engineering from the Tokyo Institute of Technology, Tokyo, Japan. In 1993 he joined the Department of Mechatronics, School of Engineering, Tokyo University of Technology, and in 2008 he moved to the university's School of Computer Science, where he is currently a professor. His research interests include applications of control theory, repetitive control, process control, Internet-based engineering education, and robotics.

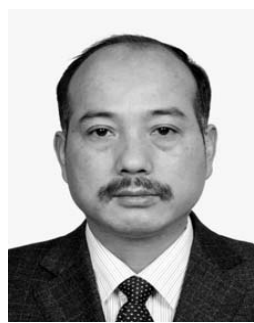

Shaowu Zhou received the B.Sc. degree in 1984 from the Chongqing University of Architectural and Civil Engineering, Chongqing, China, an M.Sc. degree in 1990 from Central South University, Changsha, and the Ph.D. degree in control science and engineering from Hunan University in 2005. Since 1990, he has been a faculty member at the Hunan University of Science and Technology, where he is currently a professor of automatic control with the School of Information and Electrical Engineering. His research interests include the application of control theory, process control, intelligent algorithm, and robotics.

Received: 7 March 2013

Revised: 12 August 2013

Re-revised: 16 December 2013 